OPEN ACCESS

Edited by:

Colin Roger MacKenzie,

National Research Council

Canada, Canada

Reviewed by:

Harald Kolmar,

Technische Universität

Darmstadt, Germany

Ellen Goldman,

United States Naval Research

Laboratory, United States

*Correspondence:

Christian Cambillau cambillau@afmb.univ-mrs.fr;

Heidi Rommelaere

Heidi.Rommelaere@ablynx.com

tPresent address:

Michael Saunders,

Christophe Blachetot and,

Hans de Haard,

argenx BVBA, Ghent, Belgium;

Geertrui Denecker,

Center for Medical Genetics

Ghent (CMGG), Cancer Research

Institute Ghent (CR/G), Medical

Research Building (MRB),

Ghent, Belgium

*These authors have contributed equally to this work.

Specialty section:

This article was submitted to Vaccines and Molecular Therapeutics,

a section of the journal

Frontiers in Immunology

Received: 30 April 2017

Accepted: 11 July 2017

Published: 21 August 2017

Citation:

Desmyter A, Spinelli S, Boutton C,

Saunders $M$, Blachetot $C$,

de Haard H, Denecker G, Van Roy M,

Cambillau $\mathrm{C}$ and Rommelaere $\mathrm{H}$

(2017) Neutralization of Human

Interleukin 23 by Multivalent

Nanobodies Explained by the

Structure of Cytokine-Nanobody

Complex.

Front. Immunol. 8:884.

doi: 10.3389/fimmu.2017.00884

\section{Neutralization of Human Interleukin 23 by Multivalent Nanobodies Explained by the Structure of Cytokine-Nanobody Complex}

\author{
Aline Desmyter ${ }^{1,2 \ddagger}$, Silvia Spinelli ${ }^{1,2 \neq}$, Carlo Boutton ${ }^{3}$, Michael Saunders $^{3 \dagger}$, \\ Christophe Blachetot ${ }^{3 t}$, Hans de Haard ${ }^{3 t}$, Geertrui Denecker ${ }^{3 \dagger}$, Maarten Van Roy ${ }^{3}$, \\ Christian Cambillau ${ }^{1,2 *}$ and Heidi Rommelaere ${ }^{3 *}$ \\ 'Architecture et Fonction des Macromolécules Biologiques (AFMB), UMR 7257, Centre National de la Recherche \\ Scientifique (CNRS), Marseille, France, ${ }^{2}$ Architecture et Fonction des Macromolécules Biologiques (AFMB), UMR 7257, \\ Aix-Marseille Université, Marseille, France, ${ }^{3}$ Ablynx N.V., Ghent, Belgium
}

The heterodimeric cytokine interleukin (IL) 23 comprises the IL12-shared p40 subunit and an IL23-specific subunit, p19. Together with IL12 and IL27, IL23 sits at the apex of the regulatory mechanisms shaping adaptive immune responses. IL23, together with IL17, plays an important role in the development of chronic inflammation and autoimmune inflammatory diseases. In this context, we generated monovalent antihuman IL23 variable heavy chain domain of llama heavy chain antibody $\left(\mathrm{V}_{\mathrm{HH}}\right)$ domains (Nanobodies ${ }^{\circledR}$ ) with low nanomolar affinity for human interleukin (hIL) 23. The crystal structure of a quaternary complex assembling hlL23 and several nanobodies against $\mathrm{p} 19$ and $\mathrm{p} 40$ subunits allowed identification of distinct epitopes and enabled rational design of a multivalent IL23-specific blocking nanobody. Taking advantage of the ease of nanobody formatting, multivalent IL23 nanobodies were assembled with properly designed linkers flanking an antihuman serum albumin nanobody, with improved hIL23 neutralization capacity in vitro and in vivo, as compared to the monovalent nanobodies. These constructs with long exposure time are excellent candidates for further developments targeting Crohn's disease, rheumatoid arthritis, and psoriasis.

Keywords: interleukin 23, nanobody, multivalent binder, crystal structure, anti-inflammatory

\section{INTRODUCTION}

By searching sequence databases for members of the IL6 cytokine family (1), a new protein, designated interleukin (IL) 23p19 was identified. This new protein had no biological activity, but formed in combination with the p40 subunit of IL12 a novel heterodimeric cytokine named IL23. The p40 subunit is shared with IL12, where it forms a heterodimer with another partner p35 (2). The human IL23-specific p19 subunit is a 189 amino acid polypeptide that contains five cysteine residues and no glycosylation sites. The p19 subunit shows an overall sequence identity of $\sim 40 \%$ to the p35 subunit of IL12. The identical p40 subunit of both cytokines binds to the receptor (R) IL12R $\beta 1$, the p35 subunit of IL12 binds to the IL12R $\beta 2$ subunit, and the p19 subunit of IL23 binds to the unique IL23R subunit. The IL23 transmembrane receptor belongs to the class I cytokine receptor family, albeit that it lacks

Abbreviations: $\mathrm{V}_{\mathrm{HH}}$, variable heavy chain domain of llama heavy chain antibody; $\mathrm{CDR}$, complementarity-determining region; $\mathrm{Nb}$, nanobody; SPR, surface plasmon resonance; $K_{\mathrm{d}}$; equilibrium dissociation constant; $k_{\mathrm{on}}$, association rate constant; $k_{\mathrm{off}}$, rate constant; rmsd, root mean square deviation; GS linker, glycine-serine linker. 
the characteristic membrane-proximal fibronectin type III-like domains. The human IL23 receptor chains are predominantly coexpressed on activated and memory T cells and NK cells, but also at low levels on monocytes, macrophages and dendritic cell (DC) populations. IL23 binds to and signals through the heterodimeric IL12R $\beta 1 /$ IL23R complex, which is associated with Tyk2 and Jak2, respectively. Upon Jak2-mediated phosphorylation of tyrosine residues located in the intracellular domain of the IL23R subunit, Stat 3 molecules are phosphorylated in turn. Phospho-Stat 3 proteins homodimerize and translocate into the nucleus, inducing transcription of cytokines such as IL17A, IL17F, IL22, and IFN- $\gamma$.

Similar to IL12, IL23 is expressed predominantly by activated DCs and phagocytic cells. IL23 is also produced by antigenpresenting cells and promotes the expansion and survival of a distinct lineage of T cells, Th17 (3). IL17, a proinflammatory cytokine predominantly produced by activated $\mathrm{T}$ cells (by Th17 cells), enhances T-cell priming and stimulates fibroblasts, endothelial cells, macrophages, and epithelial cells to produce multiple proinflammatory mediators, including IL1, IL6, TNF- $\alpha$, NOS2, metalloproteases, and chemokines, resulting in the induction of inflammation (4-6). IL17 expression is increased in patients with a variety of allergic and autoimmune diseases, such as rheumatoid arthritis, multiple sclerosis, inflammatory bowel disease, and asthma, suggesting the contribution of IL17 to the induction and/or development of such diseases. Th17/ ThIL17 cells are likely to play critical roles in the development of autoimmunity and allergic reaction, and the IL23/IL17, but not IL12/IFN- $\gamma$, axis is critical for the development of autoimmune inflammatory diseases (7).

Our aim was to generate nanobody constructs able to neutralize human interleukin (hIL) 12 and/or hIL23, which could be drug candidates for treatment of Crohn's disease, rheumatoid arthritis, or psoriasis. In this report, we describe the generation and characterization of nanobodies against p40 and the human (h) IL23specific p19 subunit. Binding and neutralization characteristics of four selected monovalent nanobodies were determined via biochemical and in vitro cell assays. As nanobodies have proven to be valuable tools for crystallization purposes in academic contexts $(8,9)$ as well as in biotechnological or biopharmaceutical contexts $(10,11)$, crystal structures of hIL23 in complex with three of those four nanobodies were generated. The structure of this quaternary complex helped to understand the high binding efficiency and blocking capacity of the nanobodies. As a result, we were able to rationalize the construction of multivalent nanobodies, whereby the two anti-p19 nanobodies were linked to improve potency and hooked up to an antihuman serum albumin (HSA) nanobody to increase the exposure time (12). The multivalent nanobodies displayed significant enhanced in vitro potency in neutralizing IL23 and proved to be very effective in an in vivo splenocyte assay performed in mice; hence, they are promising drug candidates.

\section{MATERIALS AND METHODS}

\section{IL23 Materials}

Human interleukin 23 was purchased from R\&D Systems Inc. and from eBioscience. hIL12, mouse (m) IL23, hIL23 receptor
(R)-fragment crystallizable region (Fc), and IL12R $\beta$-Fc chimera were purchased from R\&D Systems Inc. Cynomolgus monkey (cyno) IL23 was produced at Ablynx, Ghent/Zwijnaarde, Belgium. hIL23 (eBioscience) and hIL12 (R\&D Systems Inc.) were biotinylated using Sulfo-NHS-LC-Biotin (Pierce ${ }^{\mathrm{TM}}$ ).

\section{Immunization, Library Construction, and Selection of Nanobodies Directed toward hIL23}

Two Lama glamas were injected with recombinant hIL23, and two llamas received a cocktail of proteins containing a.o. recombinant hIL23 and hIL12. Each animal received seven doses of intramuscular injected antigen at weekly intervals, as described by Roovers et al. $(13,14)$. Pre-immune and immune sera were collected at day 0 , and after 3 and 6 weeks of immunization. The immune response in each animal was monitored by titration of serum samples on coated hIL12 or hIL23.

RNA was prepared from peripheral blood mononuclear cells (PBMCs) isolated from sera and lymph node biopsies. Library constructions were performed as described previously $(13,14)$, amplifying the variable heavy $\left(\mathrm{V}_{\mathrm{H}}\right)$ chain domains of heavy chain antibody $\left(\mathrm{V}_{\mathrm{HH}}\right)$ genes and ligating them into the phagemid vector pAX51 in frame with a C-terminal c-myc and hexa-histidine tag for display on phage.

After superinfection of the Escherichia coli TG1 library clones with helper phage, the presence of pAX51 allows for the production of phage particles displaying the individual nanobodies as a fusion protein with the pIII protein. Nanobodies recognizing specifically the p19 subunit of hIL23 were retrieved, allowing phages bind to coated hIL23 ( 5 or $0.5 \mathrm{nM}$ ) on microtiter plates. Phages were counter selected three times by binding to wells coated with $5 \mu \mathrm{g} / \mathrm{ml}$ hIL12 to remove p40-binding phages, and further preincubated with $1 \mu \mathrm{M}$ hIL12 in solution before adding to the hIL23 coated wells. Phages were specifically eluted with trypsin (in the case of 124C4) or with $5 \mathrm{nM}$ of recombinant IL23R (in the case of 37D5). Nanobodies recognizing specifically the p40 subunit of hIL23 and hIL12 were obtained by trypsin elution of phages bound to coated hIL23 $(0.1 \mathrm{nM})$. Subsequently, exponentially growing E. coli TG1 cells were infected with the eluted phages, and individual clones were selected, grown in 96-deep well plates ( $1 \mathrm{ml}$ volume) and induced by the addition of isopropyl- $\beta$-D-thiogalactopyranoside (IPTG) for the production of nanobodies. Since the nanobodies are secreted into the periplasmic space, this fraction was then prepared by freeze-thawing of the bacterial pellet in a phosphate-buffered saline (PBS; $100 \mu \mathrm{l})$ solution and centrifugation to remove cell fragments.

\section{ELISA-Binding Screen}

$1 \mu \mathrm{g} / \mathrm{ml}$ of cytokine (hIL12, hIL23) was immobilized directly on microtiter plates. Free-binding sites were blocked using $4 \%$ Marvel in PBS. To this, $5 \mu \mathrm{l}$ of nanobody containing periplasmic extracts in $100 \mu \mathrm{l} 2 \%$ Marvel PBS with Tween 20 were added. Nanobody binding was revealed using a mouse-anti-myc primary antibody, and a horseradish peroxidase-conjugated goat anti-mouse secondary antibody. 


\section{AlphaScreen-Based Receptor-Blocking Assay}

To determine the capacity of the nanobodies to inhibit the IL23/ IL23R or IL12/IL12R $\beta 1$ interaction, a protein-based competition was used. First, periplasmic extracts were screened for the presence of neutralization capacity at 1 dilution (25-fold), and therefore preincubated with $3 \mathrm{nM}$ biotinylated hIL23 or hIL12. Second, the potency of the neutralizing nanobodies was determined by using serial dilutions of purified p19 blocking (p19+) nanobodies (from $250 \mathrm{nM}$ to $1 \mathrm{pM}$ ) or of p40 blocking (p40+) nanobodies (from $250 \mathrm{nM}$ to $1 \mathrm{pM}$ ) preincubated with $500 \mathrm{pM}$ biotinylated hIL23 and with $3 \mathrm{nM}$ biotinylated hIL12, respectively. To these mixtures, IL23R, respectively IL12R $\beta 1$ acceptor beads (receptors bound via an antihuman Fc monoclonal antibody) and the streptavidin-coated donor beads (Perkin Elmer Inc)-were added, and further incubated for $1 \mathrm{~h}$ at room temperature. Fluorescence was measured using the EnVision Multilabel Plate Reader (Perkin Elmer Inc.) using an excitation wavelength of $680 \mathrm{~nm}$ and an emission wavelength of $520 \mathrm{~nm}$. Decrease in the AlphaScreen signal indicated that binding of biotinylated hIL23 to the IL23R or hIL12 to the IL12R $\beta 1$ is blocked by the nanobody.

\section{Surface Plasmon Resonance (SPR)}

Surface plasmon resonance was performed using a Biacore T100 instrument. hIL23 was covalently bound to a CM5 sensor chip surface via amine coupling using EDC/NHS for activation and $\mathrm{HCl}$ for deactivation. Nanobody binding was assessed using periplasmic extracts diluted $1 / 10$ for off-rate determination, and using purified nanobodies at concentrations ranging from 1 to $300 \mathrm{nM}$ for $K_{\mathrm{d}}$ determination. Each nanobody was injected for $4 \mathrm{~min}$ at a flow rate of $45 \mu \mathrm{l} / \mathrm{min}$ to allow binding to chip-bound antigen. Binding buffer without nanobody was then passed over the chip at the same flow rate to allow spontaneous dissociation of bound nanobody. $k_{\text {off }}$ values were calculated from sensorgrams obtained for the different nanobodies, and $k_{\text {on }}$ and $K_{\mathrm{d}}$ values were calculated from sensorgrams for the purified nanobodies. For binning of nanobodies, hIL23 was captured via a p40-binding nanobody, which was immobilized on a chip. After binding of one of the lead nanobodies at $500 \mathrm{nM}$, RU levels were determined to evaluate whether their levels had increased after the flowing over of the other lead nanobody at $500 \mathrm{nM}$.

\section{Cloning, Expression, and Purification of Anti-IL23 Nanobodies}

Nanobody genes were subcloned into the pAX55 expression vector in frame with a N-terminal ompA sequence, and a C-terminal c-myc and hexa-histidine tag (Ablynx; described in WO2008043821). Multivalent nanobody constructs, as outlined in Table 1 (D), were made in the pAX55 vector. They comprise of one or two anti-p19 nanobody building blocks and one building block corresponding to an anti-HSA nanobody building block (ALB1). The individual building blocks were fused by Gly/Ser linkers: 9GS (GGGGSGGGS) or 15GS (GGGGSGGGGSGGGGS). Nonsuppressor E. coli TG1 cells (Stratagene Corp.) transformed with the appropriate vector were grown at $37^{\circ} \mathrm{C}$ in Terrific Broth medium supplemented with $100 \mu \mathrm{g} / \mathrm{ml}$ kanamycin and $0.1 \%$ glucose for
3 h until optical density $\left(\mathrm{OD}_{600}\right)$ reached $\sim 4$. Nanobody expression was induced by the addition of $1 \mathrm{mM}$ IPTG, and growth of the cells was continued for $3-4 \mathrm{~h}$ at $37^{\circ} \mathrm{C}$. The periplasmic fraction was prepared according to the methods of Skerra and Plückthun (15), and His-tagged nanobodies were purified by immobilized metal affinity chromatography on a $1 \mathrm{ml} \mathrm{Ni-NTA}$ column.

For crystallization purposes, eluted fractions in $250 \mathrm{mM}$ imidazole were concentrated on an Amicon-Ultra $10 \mathrm{kDa}$ cutoff concentrator prior to being loaded on to a HiLoad 10/30 Superdex75 gel filtration column in Dulbecco's PBS (dPBS; Invitrogen). Protein concentration of the nanobodies was determined by UV spectrometry from the absorbance at $280 \mathrm{~nm}$, using their calculated extinction coefficient. For the nanobodies that had to be tested in the splenocyte assays, lipopolysaccharide (LPS) removal was performed by ion exchange in flow-through mode or by gel filtration in the presence of octylglucosylpyranoside; remaining LPS levels were determined using a limulus amebocyte lysate assay.

\section{${ } \mathrm{C}_{50}$ Determination in a Mouse Splenocyte Assay}

Spleens of five C57BL/6 mice were removed, splenocytes were harvested, and a single cell suspension was prepared. Splenocytes were washed three times in RPMI supplemented with $10 \%$ fetal bovine serum, $1 \%$ pen/strep, $80 \mu \mathrm{M} \beta$-mercaptoethanol, and $1 \mathrm{mM}$ sodium pyruvate. This medium is further referred to as complete RPMI. The splenocyte suspension was treated with $1 \times$ erythrocyte lysis buffer (155 $\mathrm{mM} \mathrm{NH}_{4} \mathrm{Cl}, 10.9 \mathrm{mM} \mathrm{KHCO}_{3}, 1.3 \mathrm{mM}$ EDTA) to remove all residual erythrocytes. After three wash steps in complete RPMI, the cells were filtered over a $100 \mu \mathrm{M}$ cell strainer and resuspended in complete RPMI containing $20 \mathrm{ng} / \mathrm{ml}$ recombinant mouse interleukin (mIL) 2 (R\&D Systems Inc.). Cells were seeded at 400,000 cells/ well in 96-well flat bottom plates. Serial dilutions of the nanobodies were preincubated with recombinant hIL23 (eBioscience) in culture medium for $30 \mathrm{~min}$ at room temperature and then incubated for a further 5 days with the splenocytes, at a final concentration of 19 pM hIL23. Supernatants were collected, and levels of mIL22 measured using ELISA (mIL22 ELISA construction kit, Antigenix America, NY, USA). All tests were done in triplicate.

\section{Acute In Vivo Splenocyte Model}

Nanobodies were injected subcutaneously (s.c.) into C57Bl/6 mice $(n=4) 24 \mathrm{~h}$ before the first of three subsequent intraperitoneal (i.p.) injections of $3 \mu \mathrm{g}$ hIL23 (at times 0,7 , and $23 \mathrm{~h}$ ). Test items were administered at a 16-fold, 3.2-fold, or 0.64 -fold molar excess to each injection of hIL23. After $31 \mathrm{~h}$, mice were sacrificed and spleens removed. Splenocytes were prepared and mIL22 measured as described above.

\section{hIL12-Dependent Proliferation of PHA Blasts}

PHA blasts were derived from cultured PBMC by stimulation with phytohemagglutinin. They were stimulated for $48 \mathrm{~h}$ with $300 \mathrm{pg} /$ $\mathrm{ml} \mathrm{hIL12.} \mathrm{The} \mathrm{cells} \mathrm{were} \mathrm{pulsed} \mathrm{with} 1 \mu \mathrm{Ci} /$ well ${ }^{3} \mathrm{H}$-thymidine for the last $6 \mathrm{~h}$, and the incorporation of ${ }^{3} \mathrm{H}$-thymidine was determined by scintillation counting in the presence of serial dilutions of nanobodies over a range of $10-10^{-5} \mu \mathrm{g} / \mathrm{ml}$. 
TABLE 1 | Interactions of the various nanobodies constructs with ILs.

(A) Nanobody types identified at screening

\begin{tabular}{|c|c|c|c|c|}
\hline & p19 binder = p19- & p19 blocker = p19+ & p40 binder $=$ p40 - & p40 blocker $=$ p40 + \\
\hline Binding to hlL23 & + & + & + & + \\
\hline Binding to hIL12 & - & - & + & + \\
\hline hIL23/IL23R blocking & - & + & - & - \\
\hline hIL12/IL12R $\beta 1$ blocking & - & - & - & + \\
\hline \multicolumn{5}{|c|}{ (B) Surface plasmon resonance experiments: $k_{\mathrm{on}}, \boldsymbol{k}_{\mathrm{off}}$, and $K_{\mathrm{d}}$ of human p19 and p40 nanobodies binding to hIL23 } \\
\hline Nanobody & Specificity & $k_{\text {off }}$ hIL23 $\left(\mathbf{s}^{-1}\right)$ & $k_{\text {on }}$ hIL23 $\left(M^{-1} \mathbf{s}^{-1}\right)$ & $K_{\mathrm{d}}$ hIL23 (M) \\
\hline 37D5 & p19+ & $1.8 \times 10^{-4}$ & $3.2 \times 10^{5}$ & $0.6 \times 10^{-9}$ \\
\hline $124 \mathrm{C} 4$ & p19- & $3.3 \times 10^{-4}$ & $1.0 \times 10^{5}$ & $3.3 \times 10^{-9}$ \\
\hline 22E11 & $\mathrm{p} 40+$ & $2.3 \times 10^{-4}$ & nd & nd \\
\hline 80D10 & $\mathrm{p} 40-$ & $9.7 \times 10^{-5}$ & $5.1 \times 10^{4}$ & $0.8 \times 10^{-9}$ \\
\hline
\end{tabular}

(C) $\mathrm{IC}_{50}$ values of the antihuman $\mathrm{p} 19$ and p40 nanobodies in AlphaScreen

\begin{tabular}{|c|c|}
\hline Human p19+ nanobody & $\mathrm{IC}_{50}(\mathrm{pM})$ hIL23 (confidence range) \\
\hline 37D5 & $110(80-170)$ \\
\hline Human p40+ nanobody & $\mathrm{IC}_{50}(\mathrm{pM}) \mathrm{hIL12}$ (confidence range) \\
\hline 22E11 & $1,300(850-1,950)$ \\
\hline
\end{tabular}

(D) Splenocyte assay (performed in triplicate)

(i) Average $\mathrm{IC}_{50}$ values of human p19 and p40 nanobodies using 19 pM hIL23 ( $\left.\pm 10 \%\right)$

(ii) Comparison of potencies of human p19 formatted, half-life extended nanobodies with 19 pM hIL23 ( $\pm 10 \%)$

\begin{tabular}{lll}
\hline Nanobody ID: P23IL & Specificity & Nanobody construct \\
\hline $\begin{array}{l}\text { (i) Monovalent } \\
\text { 37D5 }\end{array}$ & p19 blocker & 37D5 \\
22E11 & P40 blocker & 22E11 \\
(ii) Multivalent & p19 blocker & \\
0050 & p19 blocker + p19 binder & 37D5-9GS-Alb1 \\
0051 & p19 blocker + p19 binder & 37D5-9GS-Alb1-9GS-124C4 \\
0053 & p19 binder + p19 blocker & 37D5-9GS-Alb1-15GS-124C4 \\
0054 & p19 binder + p19 blocker & 37D5-15GS-Alb1-15GS-124C4 \\
0070 & p19 binder + p19 blocker & 124C4-15GS-Alb1-15GS-37D5 \\
0072 & P40 blocker + p40 binder & 22E11-9GS-Alb1-15GS-80D10 \\
0409 & 3.1 & 3.2 \\
\hline
\end{tabular}

h, human; $I C_{50}$, half maximal inhibitory concentration; IL, interleukin; nd, not determined; $R$, receptor; hIL, human interleukin. In D(ii), bold numbers emphasize the length of the GS repeats.

\section{hIL23/Nanobody Complex Purification and Characterization}

$1 \mathrm{mg}$ hIL23 in dPBS pH 7.2 was incubated with a slight molar excess of three different purified nanobodies for $1 \mathrm{~h}$ on ice. The resulting complex was separated from free nanobody excess by gel filtration chromatography on a Superdex75 column in $10 \mathrm{mM}$ HEPES pH 7.0, $150 \mathrm{mM} \mathrm{NaCl}$, and then concentrated to $7.6 \mathrm{mg} / \mathrm{ml}$ on an Amicon-Ultra filter (cutoff $50 \mathrm{kDa}$; Millipore). Mass spectrometry was performed on a matrix-assisted laser desorption/ionization time of flight (MALDI-TOF) mass spectrometer (Bruker Autoflex) according to standard procedures.

\section{Crystallization and X-Ray Diffraction}

Diffraction-quality crystals of the complex were obtained by sitting-drop vapor diffusion at $277 \mathrm{~K}$ after $4-30$ days in $16.5 \% \mathrm{PEG}$ $20 \mathrm{~K}$ and $0.1 \mathrm{M}$ MES pH 5.9. Crystals belong to the orthorhombic space group $\mathrm{P} 22_{1} 2_{1}{ }_{1}$, with unit cell dimensions: $a=102.0 \AA$, $b=134.8 \AA$, and $c=138.4 \AA$. They contain two complexes per asymmetric unit. Crystals were flash frozen to $100 \mathrm{~K}$ using $13 \%$ glycerol as the cryoprotectant. Diffraction data were collected under standard cryogenic conditions on beamline ID29, using an ADSC Quantum 4 detector at the ESRF synchrotron (Grenoble, France), processed using MOSFLM (16), and scaled with SCALA (17). The crystal structure of hIL23 in complex with three nanobodies was determined from single-wavelength native diffraction experiments by molecular replacement using PHASER (18). Refinement was performed with BUSTER (19). Figures were constructed using the PyMOL Molecular Graphics System, Version 1.5.0.4 (Schrödinger, LLC). Coordinates were deposited with the Protein Data Bank (PDB) with accession code 4GRW.

\section{RESULTS}

\section{Generation and Selection of $p 19-$ and p40-Binding Nanobodies}

To generate $\mathrm{V}_{\mathrm{H}}$ chain domains of Heavy chain antibodies $\left(\mathrm{V}_{\mathrm{HH}}\right.$ or Nanobodies ${ }^{\circledR}$ ) against the IL23 p19 and p40 subunit, llamas were immunized with recombinant hIL23. Subsequently, phage 
nanobody libraries were generated from PBMCs and lymph node biopsies (LNs) collected from these animals. This resulted in four libraries with sizes between $2 \times 10^{7}$ and $4 \times 10^{7}$, and a percentage of insert containing clones ranging from 91 to $100 \%$. After selection, nanobodies present in the periplasmic fractions of isolated clones were screened for their binding specificity to hIL12 versus hIL23 in an ELISA binding assay. Nanobodies binding to p40 were identified and recognized both hIL12 and hIL23, wheras nanobodies binding to p19 specifically recognized hIL23 only.

Periplasmic extracts were then analyzed to determine the ability of the nanobodies herein to inhibit the hIL23-hIL23R or the hIL12-hIL12R $\beta 1$ interaction via an Amplified Luminescent Proximity Homogeneous (AlphaScreen) Assay for proteinprotein interaction detection. Nanobodies decreasing the signal in this assay inhibit the hIL23/hIL23R (designated p19+) or the hIL12/hIL12R $\beta 1$ interaction (designated p40+).

Different nanobody types were identified in the binding and neutralization screening assays (Table 1, A). A selection of p19 binding (p19-), p19 blocking (p19+), p40 binding (p40-), and p40 blocking ( $\mathrm{p} 40+$ ) nanobodies were DNA sequenced. Twenty families of anti-p19 nanobody sequences were identified, with 7 families containing p19+ and 13 containing p19- nanobodies; and 24 families of anti-p40 nanobody sequences, with 12 families containing p40+ and 12 containing p40- nanobodies. The offrates of the unique family members were determined using SPR. The family members with the slowest off-rates on hIL23 or hIL12 were recloned and purified for further characterization.

\section{Affinity Determination of Anti-p19 and Anti-p40 Nanobodies}

Binding kinetics of the purified human p19+ nanobody 37D5, the p19- nanobody $124 \mathrm{C} 4$, the p40+ nanobody 22E11, and the p40- nanobody 80D10 were determined using SPR. From the sensorgrams obtained, $k_{\text {on }}, k_{\text {off, }}$ and $K_{\mathrm{d}}$ were calculated where possible (Table 1, B). The three compounds were found to display excellent off-rates for hIL23, with nanobody 37D5 having the best affinity $\left(K_{\mathrm{d}} 0.57 \mathrm{nM}\right)$. Species cross-reactivity profiles of the monovalent nanobodies were also studied using SPR. All four nanobodies bound to cynomolgus monkey (cyno) IL23 with similar off-rates, and only nanobody $124 \mathrm{C} 4$ was able to crossreact to mouse (m) IL23. Knowledge of species cross-reactivity is important in light of future animal efficacy and pharmacokinetic/ pharmacodynamic studies.

\section{In Vitro Evaluation of the Inhibiting Capacity of Anti-p19 and Anti-p40 Nanobodies}

The potency of the nanobodies to inhibit the IL23/IL23R or IL12/ IL12R $\beta 1$ interaction was determined either in a protein-based competition assay using AlphaScreen or in cell-based potency assays.

For the AlphaScreen, preincubation of a serial dilution of the p19+ nanobody 37D5 with biotinylated hIL23 reduced fluorescence intensity at $520 \mathrm{~nm}$, demonstrating that this nanobody can effectively inhibit hIL23 binding to IL23R in a dose-dependent manner with a half maximal inhibitory concentration $\left(\mathrm{IC}_{50}\right)$ of
110 pM. Preincubation of a serial dilution of the p40+ nanobody 22E11 with biotinylated hIL12 also reduced the fluorescence intensity, demonstrating that this nanobody can effectively inhibit hIL12 binding to IL12R $\beta 1$ with an $\mathrm{IC}_{50}$ of 1,300 pM (Table 1, C).

Inhibition of hIL23-mediated signaling by the nanobodies was investigated using a mouse splenocyte assay. This cell-based potency assay is based on the ability of hIL23 to stimulate mIL22 secretion from mouse spleen cells (20). The p19+ nanobody 37D5 appears to be very potent reaching an average IC50 value of 19 pM when using 19 pM hIL23 for stimulation, whereas the p40+ nanobody $22 \mathrm{E} 11$ shows an $\mathrm{IC}_{50}$ of $186 \mathrm{pM}$ (Table 1, D, i).

\section{Anti-p19 Nanobodies 37D5 and 124C4 Recognize Different Epitopes}

As shown above, nanobody 37D5 shows good neutralizing activity toward hIL23. In theory, it would be possible to further improve the hIL23 neutralization by linking nanobody 37D5 to a p19-binding non-blocking nanobody as avid interaction of such a multivalent construct with the cytokine is expected. To do so, both nanobodies need to bind to different epitopes.

An SPR experiment was conducted where hIL23 was captured via a p40-binding nanobody (80D10), which was immobilized on a chip. After binding the p19 blocker (nanobody 37D5), it was assessed as to whether a second nanobody could bind simultaneously to hIL23 p19 subunit. The interaction studies show that nanobody 37D5 can bind concurrently with the p19- nanobody 124C4, since RU levels double upon binding of the second nanobody (Figure S1 in Supplementary Material, green trace). For other p19- nanobodies tested, this was not the case (Figure S1 in Supplementary Material, non-green traces). Hence, nanobody $37 \mathrm{D} 5$ and nanobody $124 \mathrm{C} 4$ could be combined in a multivalent construct and used simultaneously for crystallization in complex with hIL23.

\section{Structure of hIL23 Bound Simultaneously to Nanobodies 37D5, 124C4, and 22E11}

The high affinity of the monovalent nanobodies and the existence of different epitopes for the p19+ nanobody 37D5, the p19nanobody $124 \mathrm{C} 4$, and the p40+ nanobody $22 \mathrm{E} 11$ encouraged us to crystallize recombinant hIL23 in complex with these three nanobodies. hIL23 was mixed with an excess of the nanobodies, and the complex was purified by gel filtration. Crystals were readily obtained, and the structure was solved by molecular replacement using the hIL23 structure [in complex with the Fab 7G10 (21)] and $\mathrm{V}_{\mathrm{HH}}$ structures $(22,23)$ as search models. The crystals contain two complexes in the asymmetric unit and the structure was refined to $R / R_{\text {free }}$ values of $18.3 \% / 21.8 \%$, respectively (Table $\mathrm{S} 1$ in Supplementary Material).

The overall structure of hIL23 with its three bound nanobodies is depicted in Figure 1. hIL23 is formed by two monomers, p19 and p40, linked by a disulfide bridge between Cys 54 (p19) and Cys177 (p40). The monomer p19 is formed by a four antiparallel helix bundle $(21,24,25)$. The p40 protein is formed of three domains, each composed of a $7 \beta$-stranded $\beta$-sandwich $(2,21,24,25)$. The interaction surface between p19 and p40 is $~ 900 \AA^{2}$ and hence quite large. The p19 monomer inserts its fourth helix between the 


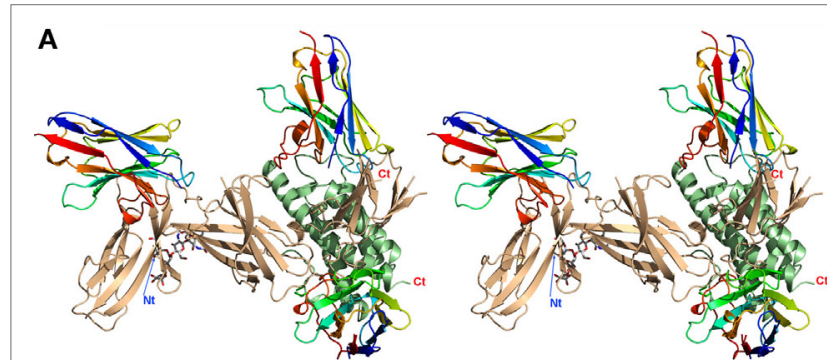

B

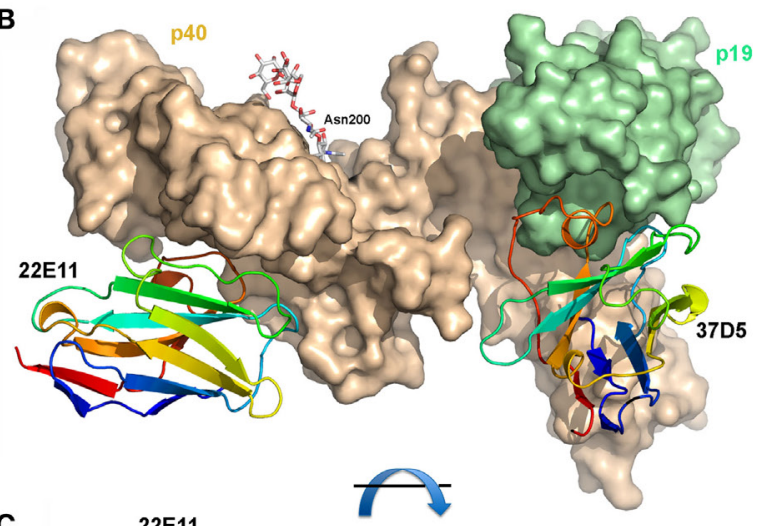

C

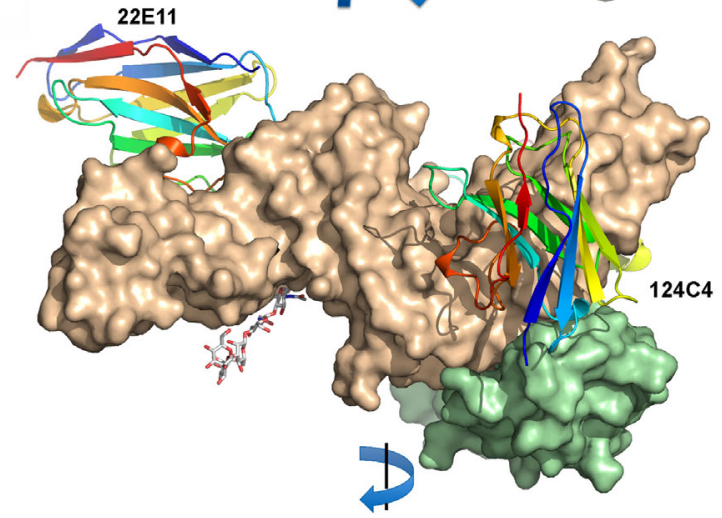

D

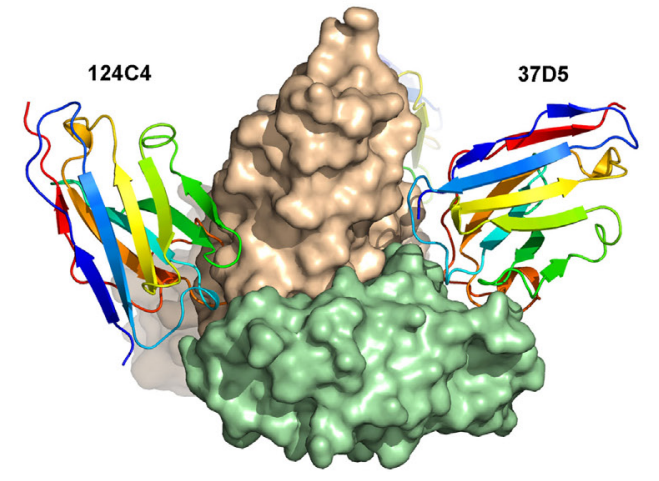

FIGURE 1 | Human interleukin (hIL) 23 in complex with three nanobodies. (A) Stereo view of hlL23 in complex with three nanobodies. p40 is shown in brown, p19 green, and the 37D5, 124C4, and 22E11 nanobodies are in rainbow colors. (B) Surface representation of hIL23, with the three nanobodies in ribbon representation. The Asn 200 branched sugar is represented by sticks. (C) $90^{\circ}$ rotation around a horizontal axis [relative to panel (B)]. (D) $90^{\circ}$ rotation around a vertical axis [relative to panel $\mathbf{( C )}$ ]. two first domains of $\mathrm{p} 40$. The interface is completed by a p19 loop joining helices $\mathrm{H} 1$ and $\mathrm{H} 2$. The structure of hIL23 in our complex is close to the structures published previously, with root mean square deviation (rmsd) values of $0.7-1.2 \AA$, mainly due to slightly different orientations of the three $\mathrm{p} 40$ domains. The rmsd values observed between the same domains in different structures are indeed much lower (0.5-0.9 $)$. Noteworthy, two saccharidic chains of the complex type are observed at Asn 200 of the two p 40 chains, with the five core sugars well defined $\left(\mathrm{GlcNAc}_{2}-\mathrm{Man}_{3}\right)$ (Figure S2 in Supplementary Material). Superposition of the two independent complexes of the asymmetric unit indicates that the two complexes are quite similar (rmsd $<1.0 \AA$ ) for p40, nanobody $124 \mathrm{C} 4$, and nanobody 22E11 (Figure S3 in Supplementary Material).

Totally unexpected from the interaction studies in solution, the structure reveals that the p19+37D5 nanobody and the p19nanobody 124C4 interact with both subunits of hIL23, p19 and p40 (Figures 1 and 2; Tables S2 and S3 and Figures S4 and S5 in Supplementary Material). As frequently observed with nanobodies (8), nanobody 37D5 and nanobody $124 \mathrm{C} 4$ bind to concave surfaces and insert their complementarity-determining regions (CDRs) in the crevices formed at the junction between p19 and p40. In contrast and as expected, the p40+22E11 nanobody interacts only with $\mathrm{p} 40$ and binds a flat surface of the cytokine's first p40 domain, remote from the two other nanobodies that bind the third $\mathrm{p} 40$ domain.

\section{Interaction of the Three Nanobodies with hIL23 and Description of a Novel Neutralizing Epitope}

The three nanobodies interact with hIL23 with high affinity, which correlates with very large buried surface areas: $\sim 1,110 \AA^{2}$ for nanobody 37D5, $\sim 830 \AA^{2}$ for nanobody $124 \mathrm{C} 4$, and $\sim 780 \AA^{2}$ for nanobody 22E11 (Table S2 in Supplementary Material). Nanobody 37D5 was initially assigned as a p19 binder and has, indeed, a larger surface of interaction with p19 $\left(850 \AA^{2}\right)$ than with p40 $\left(260 \AA^{2}\right)$ (Table S2 in Supplementary Material). It binds to the 19 helix bundle with its three CDRs and a few residues from the framework. CDR3, however, exhibits the most extended interaction with seven residues as compared to three residues for CDR1 and CDR2 (Table S3A in Supplementary Material; Figure 2B). The interaction with p40 occurs through three residues (Table S3 in Supplementary Material), with Phe 27 inserted deeply between both monomers. The $124 \mathrm{C} 4$ nanobody, which was initially assigned as a p19 binder, exhibits a binding pattern opposite to the one of 37D5, with a larger surface of interaction with p40 $\left(625 \AA^{2}\right)$ than for p19 $\left(200 \AA^{2}\right)$. Interaction with p40 involves four residues of CDR2 and seven residues of CDR3 (Table S3B in Supplementary Material; Figure 2C). Interaction with p19 involves four residues of CDR1 and one residue (Thr97) from CDR3. Finally, nanobody 22E11 interacts exclusively with p40. The interface involves a small number of contacts with CDR1 and CDR2 (two and four residues, respectively) and a large number of contacts (eight residues) from CDR3 (Table S3C in Supplementary Material; Figure 2D). 
A

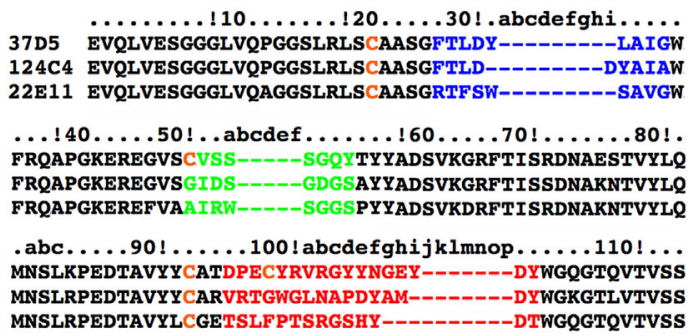

B

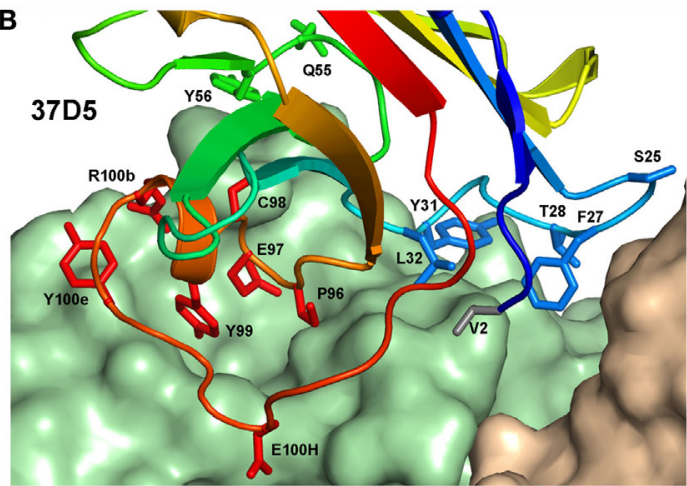

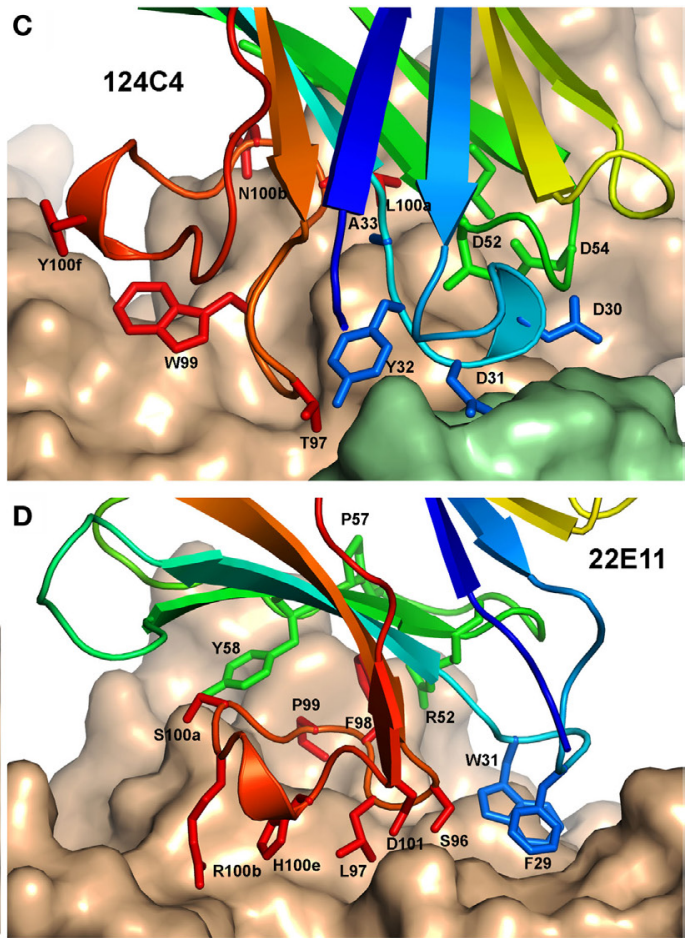

FIGURE 2 | Contacts of human interleukin (hIL) 23 in complex with three nanobodies. (A) Sequence alignment of the nanobodies 37D5, 124C4, and 22E11. Complementarity-determining region (CDR) 1 is shown in blue, CDR2 is green, CDR3 is red, and cysteines are shown in orange. (B) N-terminal, CDR, and framework 3 residues of nanobody 37D5 that interact with hIL23. (C) CDR residues of nanobody 124C4 that interact with hlL23. (D) Residues of nanobody 22E11 that interact with p40. In (B)-(D) p40 is shown in brown, p19 is green, and the 37D5, 124C4, and 22E11 nanobodies are in rainbow colors. CDR1 is shown in green, CDR2 is blue, and CDR3 is red.

\section{Formatting of the Anti-p19 Nanobodies Results in Very Potent hIL23-Neutralizing Molecules}

We generated multivalent constructs, whereby we assembled the p19-neutralizing nanobody 37D5 with the nonneutralizing nanobody $124 \mathrm{C} 4$. To prolong the half-life of the molecules for use as therapeutic agents in inflammatory diseases, a nanobody that binds serum albumin (Alb1) was included in these constructs. The human-mouse serum albumin cross-reactive nanobody Alb1 was positioned between the two anti-p19 nanobody building blocks (Figure 3). The length of the linkers fusing Alb1 to the anti-p19 nanobody building blocks was varied, based on predictions using the crystal structures. In addition, the nanobody order in the constructs was varied, as $\mathrm{N}$ - or C-terminal positioning of a certain building block can influence its binding affinity. Next to that, a half-life extended (HLE) version of nanobody 37D5 on its own (37D5-9GS-Alb1, Table 1, D, ii) was generated, since nanobody $37 \mathrm{D} 5$ is already quite potent in a monovalent format. The potency of the different constructs was determined in the mouse splenocyte assay using hIL23 as described above. The potency of 37D5-9GS-Alb1 is slightly decreased; however, potencies of the trivalent nanobodies are better (Table 1, D, ii). When placing nanobody 37D5 at the $\mathrm{N}$-terminal position, the neutralizing potency is

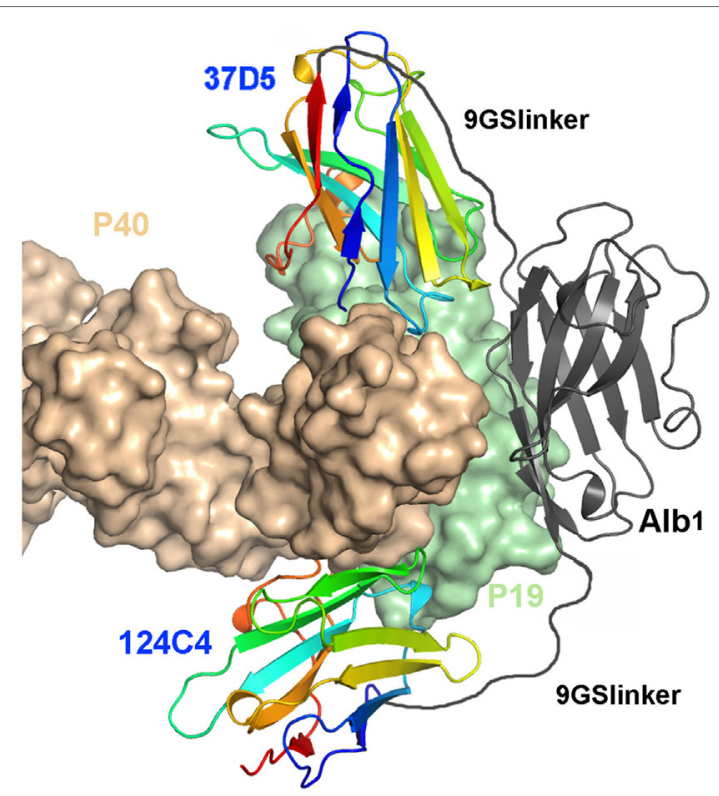

FIGURE 3 | Model of a multivalent construct combining the nanobodies 124C4 and 37D5 with the antihuman serum albumin nanobody Alb1 flanked by two 9 GS flexible linkers. This 9GS-Alb1-9GS construct is able to bridge the distance between the C-terminus of 124C4 and the N-terminus of 37D5 when bound to human interleukin 23. 
approximately fivefold better with a $9+15 \mathrm{GS}$ linker compared to a $9+9 \mathrm{GS}$ linker (Table 1, D, ii). When combining $9+15 \mathrm{GS}$ or $15+15 \mathrm{GS}$, the order of the building blocks has little influence on the potency to neutralize hIL23.

\section{HLE Multivalent Anti-p19 Nanobodies Show Improved Efficacy In Vivo}

An acute in vivo mouse splenocyte model assay demonstrated the efficacy of the formatted nanobodies P23IL0050 (37D5-9GSAlb1) and P23IL0054 (124C4-15GS-Alb1-9GS-37D5). Nanobodies were injected subcutaneously (s.c.) $24 \mathrm{~h}$ before the first of three subsequent intraperitoneal (i.p.) injections of $3 \mu \mathrm{g}$ hIL23 at times 0,7 , and 23 h occurred. Nanobodies were

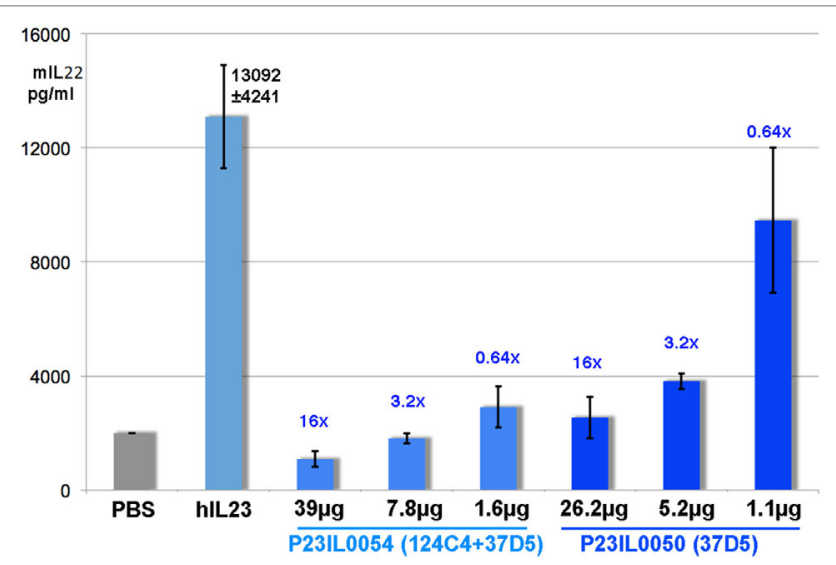

FIGURE 4 | Inhibition of human interleukin (hIL) 23 induced mouse interleukin (mIL) 22 synthesis upon administration of P23IL0050 or P23IL0054. The $y$-axis indicates the average $\mathrm{mlL} 22$ synthesis in picograms per milliliter. The $x$-axis depicts the different test groups (two nanobodies, each at three different microgram doses). Numbers in blue positioned above the bars give the molar excess ratio of nanobody administered over the $3 \mu \mathrm{g} \mathrm{hlL23}$ injected. The number above the hlL23 bar is the mean mIL22 synthesis for the control group receiving hlL23 only $(\mathrm{pg} / \mathrm{ml} \pm \mathrm{SD})$. administered at three different doses, at a 16-fold, 3.2-fold, or 0.64-fold molar excess to each injection of hIL23. P23IL0050 and P23IL0054 were capable of neutralizing hIL23 in vivo, measured as significant and complete blocking of mIL22 synthesis at the two highest doses tested (Figure 4). Administration of the lowest dose of the monovalent nanobody construct P23IL0050 (0.64-fold excess) blocked hIL23-induced mIL22 production only partially, whereas multivalent construct P23IL0054 still shows significant blocking demonstrating the power of combining different antip19 nanobodies in one construct (Figure 4).

\section{DISCUSSION}

\section{Structural Analysis of Monovalent Nanobodies}

We report here the generation and characterization of highaffinity nanobodies derived from llama heavy chain antibodies raised against hIL23. Two of the nanobodies studied revealed a high efficacy in neutralizing hIL23 in in vitro bioassays using mouse splenocytes. The $\mathrm{IC}_{50}$ values ranged from $\sim 20$ to $\sim 200 \mathrm{pM}$. To better understand the mode of action of those neutralizing nanobodies, the crystal structure of hIL23 in complex with the nanobodies 37D5 (p19+), 22E11 (p40+), and 124C4 (p19-) was generated. Comparison of our structure of hIL23, with the already known structures, i.e., hIL23 alone [3DUH (24)], hIL23 in complex with the Fab fragment (7G10) of a neutralizing antibody [3D85 (21)], and hIL23 in complex with adnectin [3QWQ (25)], shows that all structures are very similar (Figure 5). The rmsd values of p19 are between 1.0 and $1.2 \AA$, whereas those of p40 range are between 1.0 and 1.9 $\AA$ (Table S4 in Supplementary Material).

We compared the binding mode of three p19-neutralizing molecules: nanobody 37D5, Fab 7G10, and adnectin to hIL23 (Figure 5; Figure S5 in Supplementary Material). Fab 7G10 binds exclusively to p19, and the surface covered by the $V_{H}$ and variable light $\left(\mathrm{V}_{\mathrm{L}}\right)$ chains is convex, as expected from this type of binder. In contrast, both the nanobody 37D5 and adnectin bind
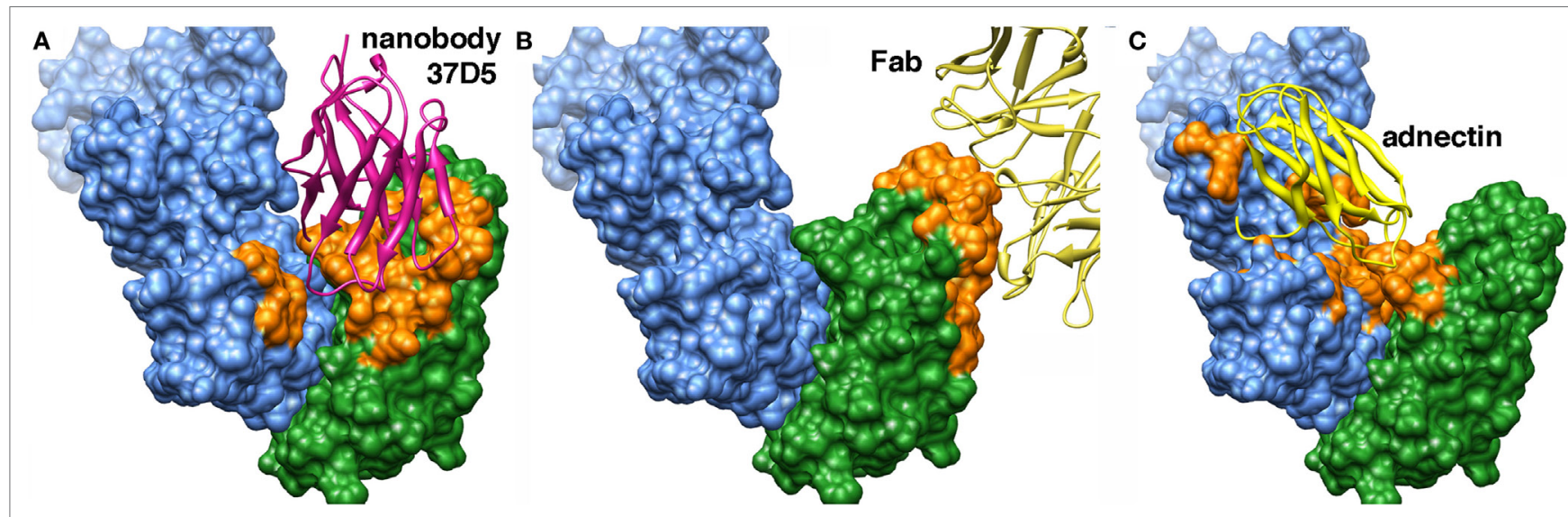

FIGURE 5 | Comparison of three complexes of human interleukin 23 with ligands. The p40 subunit is shown in blue, and p19 is green. The surface of interaction is colored orange. (A) Complex with the 37D5 nanobody (shown in pink). (B) Complex with the neutralizing Fab 7G10 (partial view; shown in yellow). (C) Complex with adnectin (shown in yellow). 
concomitantly p19 and p40. They recognize a concave surface, a result often observed for nanobodies (8). When looking at the interaction surface areas [all measured by the same server, PISA (26)], adnectin is by far covering the largest surface area $\left(1,566 \AA^{2}\right)$, followed by nanobody 37D5 (870 $\left.\AA^{2}\right)$, and Fab 7 G10 (778 $\left.\AA^{2}\right)$ (Table S5 in Supplementary Material). As those molecules are neutralizing, and hence competing with IL23R interaction, they probably overlap with the IL23R epitope. To the best of our knowledge, no structure of an IL23-IL23R complex is known to date, but some suggestions on possible IL23R epitopes have been described $(21,24)$.

To understand the fact that nanobody $124 \mathrm{C} 4$ and nanobody 37D5 were initially expected to be solely p 19 binders, but bound both p19 and p40, we superimposed our hIL23 structure on that of hIL12 [pdb $1 \mathrm{f} 45$ (2)]. IL12 has the p40 subunit in common with IL23 and forms a heterodimer with a p35 subunit, instead of p19 in IL23. There is a good overlap between the two p40 subunits and also the secondary structure elements of p19 overlap well with p35 (backbone rmsd $=1.6 \AA$ ). However, since p35 is larger than p19, some exposed regions of p40 in hIL23 are buried in hIL12. Indeed, due to the larger size of p35 versus p19, the binding mode of nanobody 124C4 to IL23 is not compatible with the p35 position and surface in IL12 (Figure S4 in Supplementary Material).

The 22E11 nanobody binds the N-terminal domain of the $\mathrm{p} 40$ monomer. Interestingly, its binding area overlaps with that of the Fab of Ustekinumab (Figure S6 in Supplementary Material; PDB: $3 \mathrm{HMX})(27,28)$. The antibody Ustekinumab targets p40, neutralizes both IL12 and IL23, and is marketed for treatment of psoriasis. Consistent with the overlap in epitopes, the anti-p40 blocking nanobody 22E11 is indeed able to neutralize hIL12 in addition to hIL23, as it could block hIL12-dependent proliferation of PBMCs stimulated with phytohemagglutinin (so called PHA blasts) with an $\mathrm{IC}_{50}$ of $95 \mathrm{pM}$ when $4 \mathrm{pM}$ hIL12 was used for stimulation (data not shown). Although the 22E11 nanobody is not as potent as Ustekinumab in neutralizing hIL23, it can be presumed that fusing nanobody 22E11 with an anti-p40 nanobody, recognizing an epitope covering the Ustekinumab p40-binding region not overlapping with 22E11, will significantly improve potency. This hypothesis is supported by a multivalent construct consisting of nanobody 22E11 and a p40 nanobody (nanobody 80D10), recognizing a non-neutralizing epitope. This construct (22E11-9GS-Alb1-15GS-80D10) improves the potency of the monovalent $22 \mathrm{E} 11$ by 300 -fold ( $\mathrm{IC}_{50}$ of $0.6 \mathrm{pM}$ in the splenocyte assay, Table 1, D, ii).

However, it is preferred to target $\mathrm{p} 19$, and hence being specific for IL23, as targeting IL12 could potentially lead to infectionrelated side effects.

\section{Use of Multivalent Nanobodies to Improve Efficacy}

Several multivalent constructs were engineered by flanking Alb1 by a linker on each side, followed by 37D5 or 124C4 on either side, in an attempt to improve the potency/efficacy of the 37D5 nanobody. As control 37D5, fused to the anti-HSA nanobody Alb1, was used. Analysis of the X-ray structure of hIL23 in complex with nanobody 37D5 and nanobody 124C4 was used to predict the peptide linker lengths needed to combine these two nanobodies in a multivalent construct, enabling simultaneous binding of both nanobodies to hIL23. The direct distance between the C-terminus of nanobody 37D5 and the N-terminus of nanobody $124 \mathrm{C} 4$ is $\sim 85 \AA$, indicating that at least a $25 \mathrm{GS}$ linker is needed in a bivalent construct. As the Alb1 nanobody was introduced between the anti-p19 nanobodies, it served as part of the linker and was flanked by two 9-mer linkers or a 9- and 15GS linker. Based on protein modeling we concluded that although a (-9GS-Alb1-9GS-) linker might be sufficient to permit binding of both nanobodies, a (-9GS-Alb1-15GS-) provides more flexibility to the construct allowing the Alb1 nanobody to accommodate better with respect to the 19 and $\mathrm{p} 40$ units. This flexibility seemed to be important since experimentally, when Alb1 was flanked by a 9- and $15 \mathrm{GS}$ linker, the $\mathrm{IC}_{50}$ value in the splenocyte assay for hIL23 was significantly better than that obtained with two 9GS linkers. Furthermore, when having $9+15$ GS or $15+15$ GS, the potency became independent of nanobody orientation, with values between 3.2 and 3.8 pM (Table 1, D, ii).

In vivo, the multivalent construct P23IL0054 (124C4-15GSAlb1-9GS-37D5) was more efficient in neutralization of administrated hIL23 to mice (Figure 4), than the monovalent p19 blocker 37D5 fused to Alb1, confirming the power of formatting nanobodies. In addition, P23IL0054 is presumably specific for IL23, unless a new heterodimeric cytokine using p19 in conjunction with another subunit emerges.

In conclusion, the multivalent approach designed and reported here has two exquisite beneficial effects. First, formatting leads to avid binding to hIL23, leading to a 10 -fold increase in potency, compared to the monovalent p19 blocker. Second and as important, by acting on two different epitopes, chances to retain the specific binding to the heterodimeric hIL23 are increased, avoiding undesired cross-reactivity with other cytokines (known or still unknown) sharing one of its components. The formatting power of the nanobody platform, together with a long in vivo residence time and low immunogenicity profile, raises the opportunity for the best of these nanobody constructs to become excellent drug candidates to treat inflammatory diseases.

\section{DATA DEPOSITION}

Coordinates were deposited with the Protein Data Bank with accession code 4GRW.

\section{ETHICS STATEMENT}

Animal work was conducted according to the guidelines set out in European Economic Community council directive 86/609/ EEC, with authorization from the prefecture of Doubs and the Department of Veterinary Services.

\section{AUTHOR CONTRIBUTIONS}

$\mathrm{HH}, \mathrm{MS}, \mathrm{HR}$, and CC designed the experiments. AD, SS, and CC purified, crystallized, and solved the X-ray structures. $\mathrm{CB}$ analyzed the X-ray structures and identified molecular interactions. $\mathrm{GD}, \mathrm{ChB}$ and $\mathrm{HR}$ were responsible for nanobody generation and 
characterization. All the authors contributed to the analysis of the results and writing of this paper.

\section{ACKNOWLEDGMENTS}

The authors would like to acknowledge help from João Viera for phage display selections; Jo Vercammen and Klara Vandriessche for screening; David Vlerick for cellular assays; Koen Van Laer for nanobody cloning and sequencing; Yvonne Mijnsbergen for purifications; and Theo Verrips for discussions. The ESRF synchrotron (Grenoble, France) is gratefully acknowledged for beamtime allocation.

\section{REFERENCES}

1. Oppmann B, Lesley R, Blom B, Timans JC, Xu Y, Hunte B, et al. Novel p19 protein engages IL-12p40 to form a cytokine, IL-23, with biological activities similar as well as distinct from IL-12. Immunity (2000) 13:715-25. doi:10.1016/ S1074-7613(00)00070-4

2. Yoon C, Johnston CJ, Tang J, Stahl M, Tobin JF, Somers WS. Charged residues dominate a unique interlocking topography in the heterodimeric cytokine interleukin-12. EMBO J (2000) 19:3530-41. doi:10.1093/emboj/19.14.3530

3. Blauvelt A. New concepts in the pathogenesis and treatment of psoriasis: key roles for IL-23, IL-17A and TGF-b1. Expert Rev Dermatol (2007) 2:69-78. doi:10.1586/17469872.2.1.69

4. Fitch E, Harper E, Skorcheva I, Kurtz SE, Blauvelt A. Pathophysiology of psoriasis: recent advances on IL-23 and Th17 cytokines. Curr Rheumatol Rep (2007) 9:461-7. doi:10.1007/s11926-007-0075-1

5. Furuzawa-Carballeda J, Vargas-Rojas MI, Cabral AR. Autoimmune inflammation from the Th17 perspective. Autoimmun Rev (2007) 6:169-75. doi:10.1016/j.autrev.2006.10.002

6. McGeachy MJ, Cua DJ. Th17 cell differentiation: the long and winding road. Immunity (2008) 28:445-53. doi:10.1016/j.immuni.2008.03.001

7. Iwakura Y, Ishigame H. The IL-23/IL-17 axis in inflammation. J Clin Invest (2006) 116:1218-22. doi:10.1172/JCI28508

8. Muyldermans S, Cambillau C, Wyns L. Recognition of antigens by singledomain antibody fragments: the superfluous luxury of paired domains. Trends Biochem Sci (2001) 26:230-5. doi:10.1016/S0968-0004(01)01790-X

9. Park YJ, Pardon E, Wu M, Steyaert J, Hol WG. Crystal structure of a heterodimer of editosome interaction proteins in complex with two copies of a cross-reacting nanobody. Nucleic Acids Res (2012) 40:1828-40. doi:10.1093/ nar/gkr867

10. Rasmussen SG, Choi HJ, Fung JJ, Pardon E, Casarosa P, Chae PS, et al. Structure of a nanobody-stabilized active state of the beta(2) adrenoceptor. Nature (2011) 469:175-80. doi:10.1038/nature09648

11. Steyaert J, Kobilka BK. Nanobody stabilization of $G$ protein-coupled receptor conformational states. Curr Opin Struct Biol (2011) 21:567-72. doi:10.1016/j. sbi.2011.06.011

12. Elsadek B, Kratz F. Impact of albumin on drug delivery - new applications on the horizon. J Control Release (2012) 157:4-28. doi:10.1016/j.jconrel.2011.09.069

13. Roovers RC, Laeremans T, Huang L, De Taeye S, Verkleij AJ, Revets H, et al. Efficient inhibition of EGFR signaling and of tumour growth by antagonistic anti-EFGR nanobodies. Cancer Immunol Immunother (2007) 56:303-17. doi:10.1007/s00262-006-0180-4

14. Roovers RC, van Dongen GA, van Bergen en Henegouwen PM. Nanobodies in therapeutic applications. Curr Opin Mol Ther (2007) 9:327-35.

15. Skerra A, Plückthun A. Assembly of a functional immunoglobulin Fv fragment in Escherichia coli. Science (1988) 240:1038-41. doi:10.1126/science.3285470

16. Leslie AG. Integration of macromolecular diffraction data. Acta Crystallogr D Biol Crystallogr (1999) 55(Pt 10):1696-702. doi:10.1107/S090744499900846X

17. Winn MD, Ballard CC, Cowtan KD, Dodson EJ, Emsley P, Evans PR, et al. Overview of the CCP4 suite and current developments. Acta Crystallogr D Biol Crystallogr (2011) 67:235-42. doi:10.1107/S0907444910045749

\section{FUNDING}

SS and CC were funded in part by Ablynx and by the Centre National de la Recherche Scientifique (France), and Aix-Marseille University (France).

\section{SUPPLEMENTARY MATERIAL}

The Supplementary Material for this article can be found online at http://journal.frontiersin.org/article/10.3389/fimmu.2017.00884/ full\#supplementary-material.

18. Bunkoczi G, Echols N, McCoy AJ, Oeffner RD, Adams PD, Read RJ. Phaser MRage: automated molecular replacement. Acta Crystallogr D Biol Crystallogr (2013) 69:2276-86. doi:10.1107/S0907444913022750

19. Blanc E, Roversi P, Vonrhein C, Flensburg C, Lea SM, Bricogne G. Refinement of severely incomplete structures with maximum likelihood in BUSTERTNT. Acta Crystallogr D Biol Crystallogr (2004) 60:2210-21. doi:10.1107/ S0907444904016427

20. Aggarwal S, Ghilardi N, Xie MH, de Sauvage FJ, Gurney AL. Interleukin-23 promotes a distinct CD4 $\mathrm{T}$ cell activation state characterized by the production of interleukin-17. J Biol Chem (2003) 278:1910-4. doi:10.1074/jbc. M207577200

21. Beyer BM, Ingram R, Ramanathan L, Reichert P, Le HV, Madison V, et al. Crystal structures of the pro-inflammatory cytokine interleukin-23 and its complex with a high-affinity neutralizing antibody. J Mol Biol (2008) 382:942-55. doi:10.1016/j.jmb.2008.08.001

22. Spinelli S, Frenken L, Bourgeois D, de Ron L, Bos W, Verrips T, et al. The crystal structure of a llama heavy chain variable domain. Nat Struct Biol (1996) 3:752-7. doi:10.1038/nsb0996-752

23. Spinelli S, Tegoni M, Frenken L, van Vliet C, Cambillau C. Lateral recognition of a dye hapten by a llama VHH domain. J Mol Biol (2001) 311:123-9. doi:10.1006/jmbi.2001.4856

24. Lupardus PJ, Garcia KC. The structure of interleukin-23 reveals the molecular basis of p40 subunit sharing with interleukin-12. J Mol Biol (2008) 382:931-41. doi:10.1016/j.jmb.2008.07.051

25. Ramamurthy V, Krystek SR Jr, Bush A, Wei A, Emanuel SL, Das Gupta R, et al. Structures of adnectin/protein complexes reveal an expanded binding footprint. Structure (2012) 20:259-69. doi:10.1016/j.str.2011.11.016

26. Krissinel E, Henrick K. Inference of macromolecular assemblies from crystalline state. J Mol Biol (2007) 372:774-97. doi:10.1016/j.jmb.2007.05.022

27. Gottlieb A, Menter A, Mendelsohn A, Shen YK, Li S, Guzzo C, et al. Ustekinumab, a human interleukin 12/23 monoclonal antibody, for psoriatic arthritis: randomised, double-blind, placebo-controlled, crossover trial. Lancet (2009) 373:633-40. doi:10.1016/S0140-6736(09)60140-9

28. Cuchacovich RS, Espinoza LR. Ustekinumab for psoriatic arthritis. Lancet (2009) 373:605-6. doi:10.1016/S0140-6736(09)60141-0

Conflict of Interest Statement: This research has been funded by Ablynx, with the exception of research by AD, SS, and CC at the Architecture et Fonction des Macromolécules Biologiques (France), whose work has been funded in part by Ablynx and the Centre National de la Recherche Scientifique (France), and the Aix-Marseille Université (France).

Copyright (c) 2017 Desmyter, Spinelli, Boutton, Saunders, Blachetot, de Haard, Denecker, Van Roy, Cambillau and Rommelaere. This is an open-access article distributed under the terms of the Creative Commons Attribution License (CC BY). The use, distribution or reproduction in other forums is permitted, provided the original author(s) or licensor are credited and that the original publication in this journal is cited, in accordance with accepted academic practice. No use, distribution or reproduction is permitted which does not comply with these terms. 